\title{
Continuous vestibular implant stimulation partially restores eye-stabilizing reflexes
}

Peter J. Boutros, ${ }^{1}$ Desi P. Schoo, ${ }^{2}$ Mehdi Rahman, ${ }^{3}$ Nicolas S. Valentin, ${ }^{3}$ Margaret R. Chow, ${ }^{1}$ Andrianna I. Ayiotis, ${ }^{1}$ Brian J. Morris, ${ }^{1}$ Andreas Hofner, ${ }^{4}$ Aitor Morillo Rascon, ${ }^{4}$ Andreas Marx, ${ }^{4}$ Ross Deas, ${ }^{4}$ Gene Y. Fridman, ${ }^{1,2}$ Natan S. Davidovics, ${ }^{3}$ Bryan K. Ward, ${ }^{2}$ Carolina Treviño, ${ }^{2}$ Stephen P. Bowditch, ${ }^{2}$ Dale C. Roberts, ${ }^{2}$ Kelly E. Lane, ${ }^{2}$ Yoav Gimmon, ${ }^{2}$ Michael C. Schubert, ${ }^{2}$ John P. Carey, ${ }^{2}$ Andreas Jaeger, ${ }^{4}$ and Charles C. Della Santina ${ }^{1,2,3}$

'Department of Biomedical Engineering and 2Department of Otolaryngology - Head \& Neck Surgery, Johns Hopkins School of Medicine, Baltimore, Maryland, USA. ${ }^{3}$ Labyrinth Devices, LLC, Baltimore, Maryland, USA. ${ }^{4}$ Med-El GmbH, Innsbruck, Austria.

Conflict of interest: CCDS, GYF, and The Johns Hopkins University (JHU) hold royalty interests in pending and awarded patents related to technologies discussed in this manuscript (AU2014216248A1,CA2786717C, US7225028B2, US7647120B2, US8751012B2, US9242094B2, US20150223683A1). CCDS holds an equity interest in and is CEO/chief scientific officer of Labyrinth Devices, LLC. The terms of this arrangement are managed in accordance with JHU policies on conflicts of interest. At the time of their contributions to this work, MR, NSV, and NSD were employees of Labyrinth Devices, LLC, and $A H, A M R, A M, R D$, and $A$ ) were employees of Med-El GmbH.

Copyright: (c) 2019, American Society for Clinical Investigation.

Submitted: February 26, 2019

Accepted: October 4, 2019

Published: November 14, 2019.

Reference information: JCI Insight.

2019;4(22):e128397.

https://doi.org/10.1172/jci.

insight.128397.
BACKGROUND. Bilateral loss of vestibular (inner ear inertial) sensation causes chronically blurred vision during head movement, postural instability, and increased fall risk. Individuals who fail to compensate despite rehabilitation therapy have no adequate treatment options. Analogous to hearing restoration via cochlear implants, prosthetic electrical stimulation of vestibular nerve branches to encode head motion has garnered interest as a potential treatment, but prior studies in humans have not included continuous long-term stimulation or 3D binocular vestibulo-ocular reflex (VOR) oculography, without which one cannot determine whether an implant selectively stimulates the implanted ear's 3 semicircular canals.

METHODS. We report binocular 3D VOR responses of 4 human subjects with ototoxic bilateral vestibular loss unilaterally implanted with a Labyrinth Devices Multichannel Vestibular Implant System vestibular implant, which provides continuous, long-term, motion-modulated prosthetic stimulation via electrodes in 3 semicircular canals.

RESULTS. Initiation of prosthetic stimulation evoked nystagmus that decayed within $\mathbf{3 0}$ minutes. Stimulation targeting 1 canal produced 3D VOR responses approximately aligned with that canal's anatomic axis. Targeting multiple canals yielded responses aligned with a vector sum of individual responses. Over 350-812 days of continuous $24 \mathrm{~h} / \mathrm{d}$ use, modulated electrical stimulation produced stable VOR responses that grew with stimulus intensity and aligned approximately with any specified $3 D$ head rotation axis.

CONCLUSION. These results demonstrate that a vestibular implant can selectively, continuously, and chronically provide artificial sensory input to all 3 implanted semicircular canals in individuals disabled by bilateral vestibular loss, driving reflexive VOR eye movements that approximately align in $3 \mathrm{D}$ with the head motion axis encoded by the implant.

TRIAL REGISTRATION. ClinicalTrials.gov: NCT02725463.

FUNDING. NIH/National Institute on Deafness and Other Communication Disorders: R01DC013536 and 2T32DC000023; Labyrinth Devices, LLC; and Med-El GmbH.

\section{Introduction}

Because it rises to the level of conscious perception only when abnormal, vestibular sensation is less familiar than the 5 senses of sight, hearing, smell, taste, and touch. Some prominent physicians and surgeons of a century ago considered the vestibular labyrinth a vestigial organ relevant to human experience only in its ability to cause disease (1). However, inner ear sensation of head motion and tilt plays essential roles in stabilizing vision, posture, gait, cerebral perfusion, and spatial orientation. Consequently, bilateral loss of vestibular sensation unleashes a panoply of symptoms, including inability to see clearly during head movement, postural instability, disequilibrium, and fatigue from exerting cognitive effort to perform normally automatic tasks, such as walking down a grocery store aisle while looking at items on the shelves (2-4). 
The impact of bilateral vestibular loss (BVL) on quality of life, productivity, and health care consumption is substantial. Sun et al. estimated that affected US adults incur a reduction in quality of life similar to that caused by severe bilateral hearing loss and a mean per capita annual economic burden of $\$ 13,000$ (range $\$ 0-\$ 49,000$ ) (3). When considered at a population level, this affliction endured by individuals extends to annual societal costs of more than $\$ 1$ billion in the United States alone in light of the 28 per 100,000 point prevalence of severe, disabling BVL among adults, or about 64,000 adults, in the United States (4). Worldwide, chronic bilateral loss of vestibular sensation afflicts an estimated 1.8 million adults. Children with congenital BVL ordinarily fare better than adults who lose function later in life (according to the authors' clinical experience), but they typically reach postural milestones, such as sitting, standing, and walking, after significant delays, and even apparently well-compensated children experience poor vision during head movement and difficulty participating in activities that come more easily to their peers with normal vestibular sensory input (5).

In contrast to the now routine and highly effective treatment of severe sensorineural hearing loss, for which cochlear implants provide prosthetic auditory sensation to nearly a million individuals worldwide and are currently implanted at a rate of more than 50,000 devices/yr (6-8), individuals with adult-onset BVL who fail to compensate despite rehabilitation therapy and cessation of vestibular suppressant medications currently have no adequate treatment options. This marked disparity of treatment options is especially striking because the anatomic and physiologic similarities between the cochlea and the vestibular labyrinth would seem to make the latter an easy target for prosthetic stimulation using a modified cochlear implant.

Whereas the cochlea decomposes sounds into their frequency components using a single neurosensory apparatus, each vestibular labyrinth divides the task of sensing head rotation, translation, and tilt among 5 distinct sensory organs, including 2 gravitoinertial acceleration sensors (the utricle and saccule) and 3 semicircular canals that each sense 1 component of $3 \mathrm{D}$ head rotational velocity (Figure 1). Each canal in the left ear is approximately coplanar with an oppositely oriented partner in the right ear. The difference in vestibular afferent neuron activity from 2 complementary canals provides an approximately linear signal encoding head rotational velocity about an axis perpendicular to their plane. The 6 canals of the 2 ears comprise 3 mutually orthogonal coplanar pairs that decompose a head rotation about any $3 \mathrm{D}$ axis into its components about the LARP, RALP, and LHRH canal plane axes, forming 3 independent and complementary streams of directional information. Those neural signals in turn drive vision- and posture-stabilizing reflexes. One of these, the 3D angular vestibulo-ocular reflex (VOR), stabilizes vision during head rotation by generating eye movements that stabilize images of Earth-fixed objects on the retinae $(9,10)$. Others integrate information from the utricles, saccules, and canals to stabilize the head and body, facilitate upright posture and gait, and support regulation of blood pressure.

Like the cochlea, the vestibular labyrinths rely on hair cells to transduce fluid movement into neural signals. Although failure of vestibular reflexes can be caused by downstream lesions in the central nervous system, it often results from hair cell injury, dysfunction, or death caused by ototoxic injury (e.g., by gentamicin and other aminoglycoside antibiotics), ischemia, infection, Ménière's disease, or genetic inner ear abnormalities (11). For many such cases, the insult spares primary vestibular afferent neurons (12). When vestibular nerve branches to the 3 canals are at least partially intact, prosthetic electrical stimulation encoding head motion should provide an effective means of partially restoring vestibular sensation and alleviating BVL symptoms. This approach is directly analogous to that of cochlear implants, except that a head motion sensor replaces the cochlear implant's microphone and electrodes are designed to deliver current to branches of the vestibular nerve rather than subsections of the cochlear nerve.

Electrical stimulation of vestibular afferent neurons has proved effective in driving reflexive eye movements in bird (13), cat (14-18), guinea pig (19-24), chinchilla (25-29), rabbit (16, 24), dog (18), and nonhuman primate $(15,18,30-35)$ animal models. Those preclinical studies provided promising evidence suggesting that a vestibular implant designed to selectively stimulate individual canal nerve branches should produce sensory percepts and VOR eye movement responses in the plane of the targeted canal. (Like the present study, animal research has focused mostly on the semicircular canals and VOR because ampullary nerves - i.e., semicircular canal branches of the vestibular nerve - are spatially distinct and uniform in directional sensitivity, whereas the utricle and saccule nerves pose a greater challenge because of dense packing of hair cells with different directional sensitivities.) Importantly, they also showed that coordinated activation of multiple vestibular nerve branches can elicit compensatory VOR eye rotations about an axis approximating the vector sum of the sensitivity axes for each canal, scaled by the relative magnitudes of 

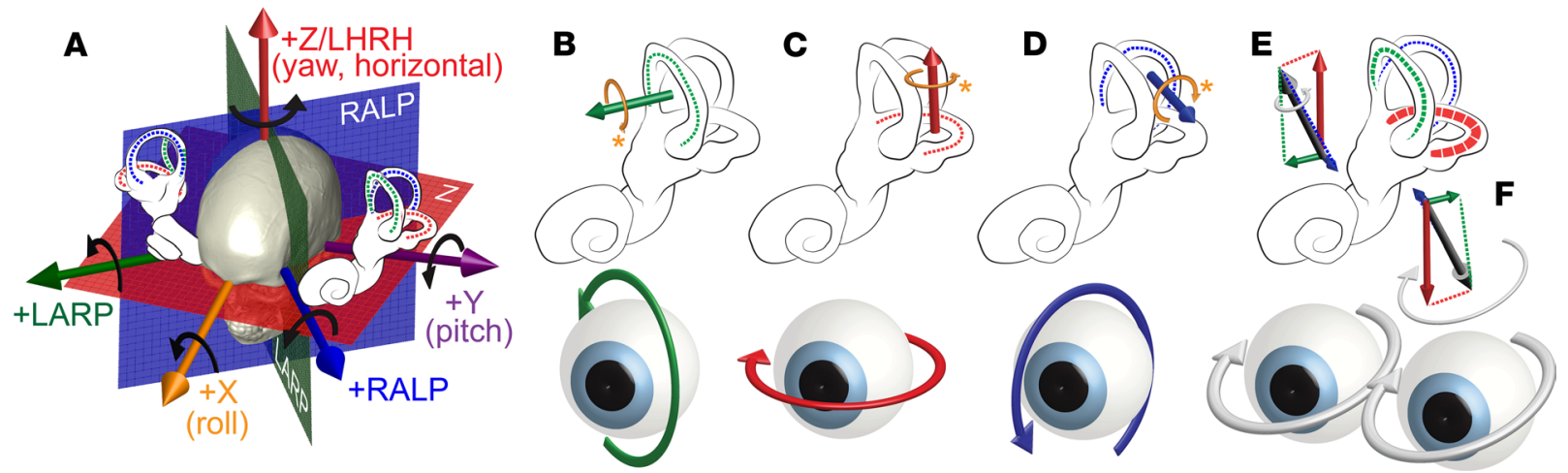

Figure 1. Coplanar pairs of semicircular canals in the vestibular labyrinths encode 3D head rotational velocity in 3 mutually orthogonal components. Relative levels of activity within vestibular nerve branches innervating a labyrinth's 3 semicircular canals encode 3 mutually orthogonal canal-aligned components of the head rotational velocity's 3D axis. The vestibulo-ocular reflex (VOR) drives eye rotations that counteract head rotation to keep images stable on the retinae. (A) Pairs of coplanar canals normally encode 3 linearly independent components of head rotational velocity about axes perpendicular to the left horizontal (LH) and right horizontal canals (LHRH or $+z$ axis), left anterior and right posterior canals (LARP), and right anterior and left posterior canals (RALP). By convention, positive rotations denote right-hand rule rotations as shown by black arrows. Each canal is most sensitive to rotation about an axis approximately perpendicular to its anatomic plane. (B-D) Head rotations about canal axes that excite the LA, LH, and LP canal, respectively, normally drive VOR responses that rotate both eyes in the opposite direction about the LARP, LHRH, and RALP axes. Physiologically excitatory directions (shown by gold arrows) are not always positive by the right-hand rule mathematical convention. (E) Head rotation about an arbitrary axis excites or inhibits each canal according to a cosine dependence on the angle between the axis of head rotation and the canal's anatomic axis. (F) Relative activity on the 3 canals in each labyrinth (and their coplanar partners in the other ear) normally drives a VOR response that helps keep images of Earth-stationary objects stable on the retinae. Without the VOR, image slip on the retinae degrades vision during quick head rotations. Although most studies of the VOR measure and describe only yaw ( $z$ /LHRH in panel A, also called "horizontal") and/or pitch ( $y /$ PITCH, also called "vertical") components, all 3 components of the $3 D$ VOR are required to maintain stable vision, and measurement of all 3 components is required to accurately estimate the relative levels of activity on each of a labyrinth's 3 semicircular canals. Reproduced by permission from Labyrinth Devices, LLC, (2019.

stimuli delivered to each canal. Animal studies using prototype devices that sense 3D head rotation and modulate electrical stimulation to ampullary nerves have demonstrated partial restoration of conjugate binocular VOR responses $(31,34,35)$, recruitment of central neuronal circuits that adaptively minimize directional misalignment $(28,32)$, and implementation of a $3 \mathrm{D}$ coordinate transformation that further reduced misalignment $(26,33)$.

Over the past decade, groups investigating prosthetic stimulation of the human vestibular labyrinth have demonstrated that stimulation targeting vestibular nerve branches can evoke eye movements consistent with target canal orientations. Starting in 2007, Guyot and colleagues described intraoperative electrical stimulation of the posterior canal ampullary nerve in 3 subjects undergoing ear surgery under local anesthesia and observed approximately vertical VOR responses (36). By 2018, that group had implanted 13 cochlear implantation candidates at Geneva University Hospital and Maastricht University Medical Center with a cochlear implant modified to allow implantation of 1-3 electrodes in semicircular canals or near ampullary nerves that innervate those canals. Responses were variable, but at least 1 electrode in each subject elicited measurable VOR responses that approximately aligned with the target canal when assayed using $2 \mathrm{D}$ video-oculography (reviewed in ref. 37).

A University of Washington (UW) group led by Rubinstein and Phillips described 4 subjects with Ménière's disease who underwent implantation of a vestibular stimulator intended to pace the labyrinth during vertigo attacks $(38,39)$. All subjects implanted in that study suffered profound postoperative loss of hearing and vestibular sensation in the implanted ear (38). Consequent cessation of Ménière's attacks rendered the planned use of a vestibular pacemaker unnecessary; however, transient electrical stimulation elicited VOR eye movements roughly aligned with the intended plane in 5 of 11 implanted canals. Responses fluctuated in subsequent transient stimulation sessions distributed over more than a year and decreased from session to session in most cases. Combined with recent evidence from nonhuman primate studies revealing that transient prosthetic electrical stimulation induces long-term depression in vestibular central neurons, suppressing VOR responses to subsequent stimulation $(40,41)$, the UW data raise pressing questions regarding feasibility of vestibular implants as a clinical treatment intended to support long-term, continuous sensory restoration.

Another question unanswered by prior studies in humans is whether prosthetic stimulation can selectively address each of the 3 ampullary nerves with sufficient specificity to accurately encode head rotation 
in 3 dimensions (3D). Although prior human studies demonstrated that transient prosthetic vestibular stimulation can produce modest electrically-evoked eye movements, they all used 2D oculographic techniques, which cannot provide adequate information to accurately estimate relative activation of the 3 canals' ampullary nerves. This is particularly important because $2 \mathrm{D}$ oculography, which measures horizontal and vertical movements but not torsional/roll eye movements, cannot distinguish between selective stimulation of the horizontal canal's ampullary nerve and nonspecific stimulation that spreads current throughout the labyrinth, equally activating all 3 ampullary nerves (14). More generally, 2D oculography fails to accurately measure $3 \mathrm{D}$ eye movements for all cases that include significant roll components. If one defines significant roll components to be those with magnitude at least $20 \%$ of the total stimulus or response magnitude, then $2 \mathrm{D}$ oculography fails to accurately describe reality for $80 \%$ of possible axes. If one defines significant roll components to be at least $10 \%$ of the total magnitude, then $2 \mathrm{D}$ oculography fails for $90 \%$ of axes.

In this report, we present $3 \mathrm{D}$ binocular oculographic data for the first 4 human subjects (MVI001MVI004) to undergo vestibular implantation and continuous, long-term, motion-modulated prosthetic stimulation. In contrast to systems used to transiently deliver stimulation in a laboratory setting in prior studies with humans, the MVI Multichannel Vestibular Implant System (MVI) developed by Labyrinth Devices, LLC, and Med-El GmbH is designed and able to continuously treat individuals suffering from severe/profound loss of semicircular canal function $24 \mathrm{~h} / \mathrm{d}$ over a lifetime of use. It therefore continuously senses 3D head rotational velocity and delivers pulse rate- and pulse amplitude-modulated electrical stimuli to the 3 ampullary nerve branches of the implanted labyrinth's vestibular nerve. The data presented offer insight into whether sustained use of vestibular implant stimulation can be effective. Specifically, we describe 3D electrically evoked vestibulo-ocular reflex (3D eeVOR) responses to initial onset of constant-rate electrical stimulation with the head motionless, to modulation of prosthetic input targeting individual canals, to coordinated stimulation of multiple canal nerve branches encoding different $3 \mathrm{D}$ axes of head rotation, and to whole-body rotation during motion-modulated and constant-rate electrical stimulation during continuous, $24 \mathrm{~h} / \mathrm{d}$ prosthetic vestibular stimulation. These data provide an opportunity to determine whether the human labyrinth's 3 ampullary nerves can be prosthetically stimulated with sufficient strength, selectivity, and safety over a long duration to drive conjugate reflex eye movements that align with the intended head rotation axis in 3 dimensions and partially restore the "sixth sense" in 3D.

\section{Results}

Overview. The implanted stimulator component of the Labyrinth Devices MVI is a Med-El Concerto cochlear implant stimulator modified for implantation in semicircular canals (Figure 2). It includes an electrode array with 9 stimulation electrodes, stimulation and recording reference electrodes, hermetically encapsulated electronics, 3 fixation magnets, and an antenna for transcutaneous inductive transmission of power and control signals from external system components. The electrode array (Figure 2, B-E) comprises 10 platinum/iridium electrodes: 3 (designated E3-E5) on a linear array implanted in the posterior canal; 2 linear arrays of 3 electrodes each, joined to form a forked array inserted into the horizontal (E6-E8) and anterior (E9-E11) ampullae; and a braided platinum/iridium wire inserted into the common crus as the reference/return electrode for stimulus currents injected via E3-E11. The implant receives power and control signals from an external HWU (Figure $2 \mathrm{~F}$ ), which magnetically couples to the scalp over the implant and continuously measures $3 \mathrm{D}$ angular head velocity using a micromachined motion sensor (Invensense MPU6050). The HWU receives power and commands from a lanyard-worn PCU (Figure 2G), which retains patient-specific device stimulation parameters, processes head movement data, dictates pulse timing, provides power to the rest of the system for up to 48 hours from a rechargeable AA-sized battery, and draws upon internal supercapacitors to maintain uninterrupted operation during battery changes.

In the system's normal, motion-modulated mode of operation, the component of head rotational velocity reported by the HWU's sensor for each canal plane modulates the pulse rate, pulse amplitude, or both on the electrode in the corresponding canal. Increasing pulse rate and/or amplitude increases vestibular primary afferent neuron firing rates, mimicking function of a normal semicircular canal during excitatory/ ipsiversive head rotation about that canal's axis. For example, to encode a $1-\mathrm{Hz}, 100^{\circ} / \mathrm{s}$ peak velocity sinusoidal head velocity waveform (Figure $2 \mathrm{H}$ ), subject- and canal-specific maps in the PCU modulated pulse rate and current amplitude (Figure 2, I-K) of charge-balanced biphasic cathodic-first pulses delivered via 1 or more electrodes to encode corresponding components of head velocity amplitude about each canal's axis of rotation. When the system is running in its usual motion-modulated mode but the head velocity 

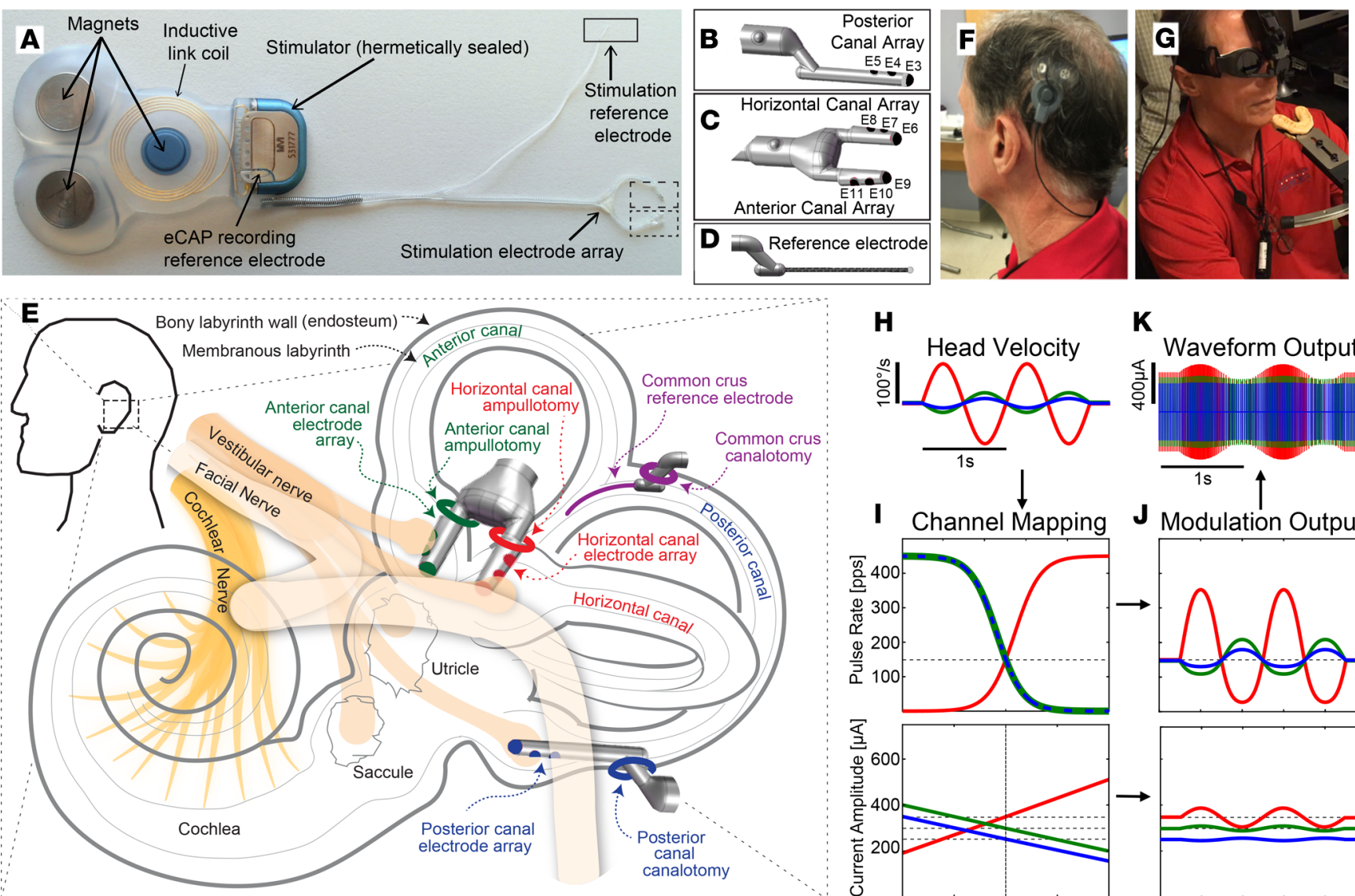

H

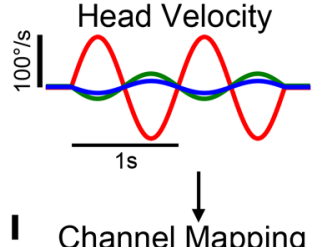

$\mathbf{K}$
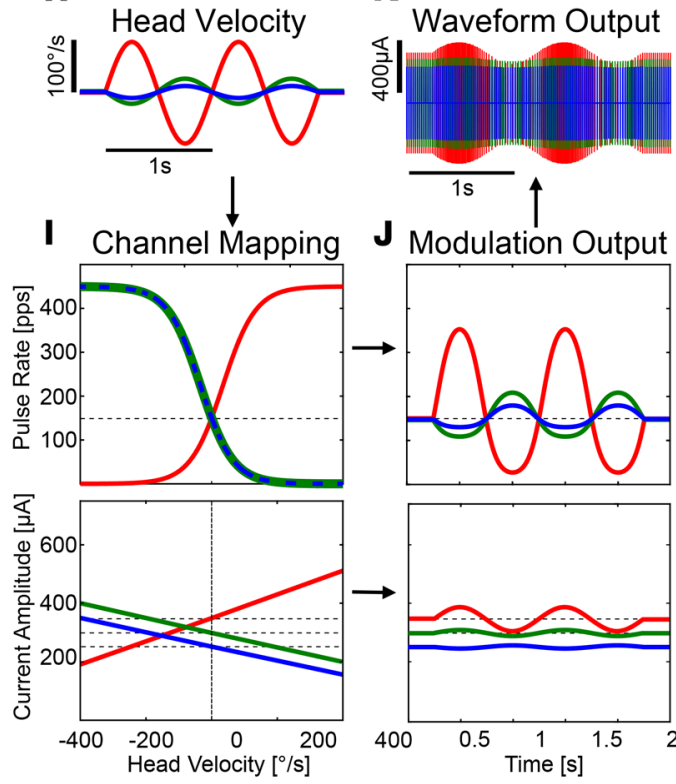

Figure 2. Overview of the Labyrinth Devices MVI and study. (A) The MVI stimulator comprises 3 fixation magnets, an inductive coil link, electrical current stimulator circuitry, stimulation electrode array, a stimulation reference electrode, and a recording reference electrode. The electrode array includes a 3-electrode shank for the (B, E3-E5) posterior canal, a forked subarray with 2 shanks for the (C, E6-E8) horizontal, and (D, E9-E11) anterior canals, and a stimulation reference electrode. (E) Surgical diagram illustrating electrode implantation sites, comprising surgical openings drilled in each of 3 canal ampullae and the common crus of 1 labyrinth. (F) The head-worn unit (HWU), magnetically coupled to subject MVI001's scalp over his implanted stimulator, houses a 3D motion sensor and inductively supplies power and control signals to the implant. (C) The power and control unit (PCU, hanging on lanyard) houses a battery and control circuity. A Labyrinth Devices 3DBinoc video-oculography system (top) records horizontal, vertical, and torsional components of 3D eye position during VOR responses to natural and/or prosthetic stimulation. (H) Example 3D head velocity waveforms (corresponding to vectors in Figure 1D) modulate MVI pulse rate and amplitude. Input waveforms can be either actual head motion sensed by the HWU or synthetically generated by MVI fitting software. (I) Example pulse rate and amplitude modulation maps for each left ear canal. Top image portrays head velocity to pulse rate map; lower plot displays maps for pulse amplitude. Maps use non-zero pulse rate and current amplitude for $0^{\circ} / \mathrm{s}$ to evoke neural activity mimicking spontaneous afferent neuron discharge (dashed lines). The MVI encodes excitatory and inhibitory head motions via coordinated up- and downmodulation of pulse rate and amplitude ( $\mathbf{J}$ and $\mathbf{K})$. (L) This is an open-label, nonrandomized, early feasibility study of applicants self-identified as potential trial candidates. Reproduced by permission from Labyrinth Devices, LLC, @2019.
L Recruited: Assessed eligibility (37)

Excluded (33)
Met exclusion criteria (11)
Declined to enroll (3)
Awaiting entry (19)
Other (0)

reported by the HWU is 0 , the implanted stimulator delivers a constant, non-zero rate and current baseline stimulus to mimic the spontaneous activity typical of a normal canal's primary afferent neurons when the head is not moving and to allow downmodulation of input to encode inhibitory head rotations $(42,43)$.

Because the brief charge-balanced pulses the implant delivers are exclusively excitatory, the system encodes inhibitory/contraversive head rotations by downmodulating pulse rate and/or amplitude from a non-zero tonic level of stimulation representing absence of head motion. This is similar to the normal canal physiology, except that although a normal canal can downmodulate ampullary nerve activity all the way to zero during a fast contraversive head rotation, downmodulation of prosthetic stimuli can decrease nerve activity only down to, but not below, the spontaneous activity of undriven vestibular afferent neurons. 
This effect limits the ability of a unilaterally implanted stimulator to encode fast contraversive head rotations. Acclimating a unilaterally implanted subject to non-zero tonic stimulation at a pulse rate well above typical spontaneous firing rates of most vestibular afferents provides adequate margin for the system to encode contraversive head rotations through downmodulation, but sudden onset of unilateral prosthetic input can be expected to cause vertigo that persists until the central nervous system adjusts to the sudden imbalance in vestibular input from the 2 ears. The intensity and duration of vertigo, nystagmus, and postural imbalance after initial onset of stimulation are likely to determine whether vestibular implant activation can be accomplished cost-efficiently on an outpatient basis (similar to cochlear implantation) or will require a hospital admission costly enough to negatively affect the cost utility of vestibular implantation as a treatment. We therefore measured nystagmus during and immediately after initial device activation.

We measured binocular 3D eye movement responses to prosthetic vestibular stimulation using Labyrinth Devices 3DBinoc video-oculography goggles, which reported 3D angular position for each eye at 100-180 frames/s. We used 3D rotational kinematic transformations to convert data to yaw, LARP, and RALP angular velocity components in semicircular canal coordinates, accounting for canal orientation relative to the HWU sensor and skull using HWU responses to standardized head rotations delivered using a Labyrinth Devices aHIT Automated Head Impulse Test motion system and 3D reconstructions of post-implantation computed tomography scans (44-48).

To examine responses driven solely by prosthetic input, we acquired data both during whole-body rotation on a rotary chair and during a virtual head rotation (i.e., prosthesis-only) stimulus paradigm in which the subject's head remained stationary on an Earth-fixed bite block while the MVI system delivered sinusoidally modulated stimuli identical to those it would normally deliver during head rotation.

Under a protocol approved by The Johns Hopkins School of Medicine Institutional Review Board and the US Food and Drug Administration, all subjects have worn their MVI systems continuously, $24 \mathrm{~h} / \mathrm{d}$, for over 1 year. This is the first and only trial to date of long-term, at-home stimulation via a vestibular implant in humans.

$3 D$ oculography reveals conjugate binocular VOR responses aligned with the implanted canal, consistent with selective activation of vestibular nerve branches. Three weeks after implantation and before onset of tonic stimulation, we tested each electrode using 20 cycles of a $2-\mathrm{Hz}$-modulated pulse train that alternated in pulse rate between 200 pulses per second (pps) and 0 pps for $200 \mathrm{~ms}$ and $300 \mathrm{~ms}$, respectively, while measuring binocular 3D VOR responses in darkness with the subject's head stationary on a bite block. Pulse amplitude was varied from the minimum current level that evoked a discernible eye movement up to the maximum level that was tolerable for the subject and maintained a consistent motion percept, determined separately for each electrode contact and pulsatile phase duration (Supplemental Table 1; supplemental material available online with this article; https://doi.org/10.1172/jci.insight.128397DS1). Maximum tolerable level was determined by slowly increasing current amplitude while the subject reported direction and intensity of a head motion percept and stopping if the subject reported tinnitus (spurious stimulation of auditory afferents), any sign of facial nerve stimulation (facial discomfort, taste disturbance, blepharospasm, or tearing), or a change in motion percept direction, signifying activation of nontarget vestibular structures.

Ideally, prosthetic stimulation delivered via an electrode targeting 1 canal when the head is stationary should elicit 3D VOR responses for both eyes that align with the target canal's anatomic axis and with each other with speed sufficient to match head angular velocity during a quick head rotation. Approximately aligned responses were observed for at least 1 electrode in every implanted canal of every subject. For example, subject MVI002's cycle-averaged binocular eeVOR responses to $100 \mu \mathrm{s} /$ phase current pulses were robust and grew monotonically with current amplitude (Figure 3, A-C). Stimuli delivered via electrode E3 in the LP canal (Figure 3A) produced the desired RALP component in both eyes, although an unintended LARP component grew in the right eye with increasing current. Stimulation via electrode E6 in the LH canal (Figure 3B) evoked almost purely horizontal eye rotation. Electrode E9 in the LA canal (Figure 3C) elicited right eye movements well aligned with the intended LARP axis and left eye movements similar in magnitude but less well aligned. The magnitude of 3D eeVOR responses and their alignment with the canal-aligned responses they are intended to elicit are sufficient to conclude that MVI stimulation can effectively and selectively activate individual ampullary nerves (Figure 3, D and E). All 3 of the other subjects had initial responses qualitatively similar to those of subject MVI002 but typically with lower magnitude or greater misalignment, particularly for stimulation targeting the LH canal, which in responses of subjects MVI003 and MVI004 were consistent with nearly equal excitation of LH and LA canals (Supplemental Figure 1). 


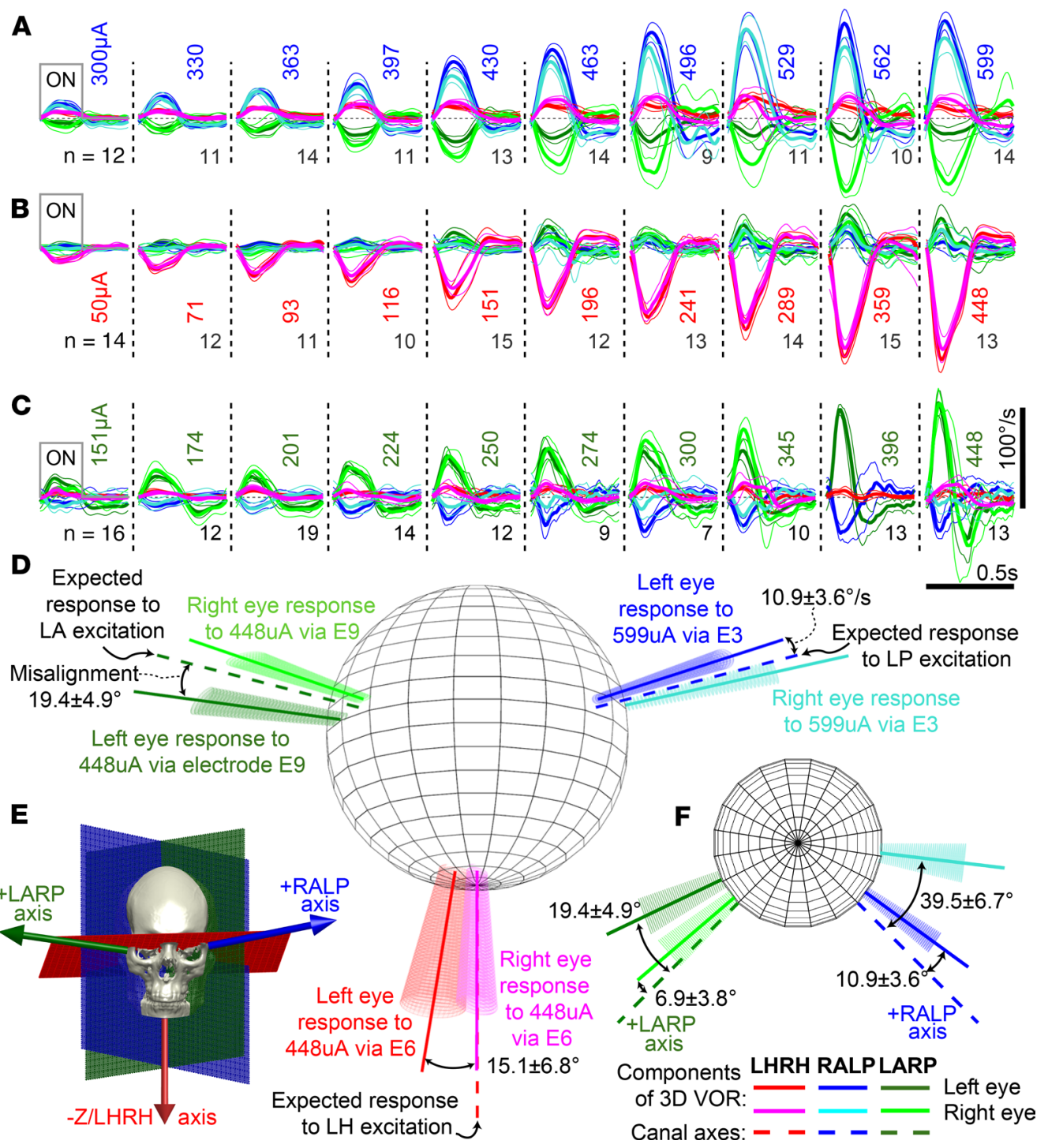

Figure 3. MVI stimulation evokes 3D VOR responses align with targeted semicircular canal. Mean \pm SD cycle-averaged binocular 3D VOR eye velocity responses of subject MVIOO2 during $n$ cycles of $2 \mathrm{~Hz}, 40 \%$ duty cycle, square-wavemodulated (200 pulses/s for $200 \mathrm{~ms}, 300 \mathrm{~ms}$ off), biphasic, charge-balanced $100 \mu \mathrm{s} / \mathrm{phase}$ current pulse trains. (A) Stimulation via electrode E3 in LP canal ampulla with pulses of 300-599 $\mu \mathrm{A}$. (B) Stimulation via electrode E6 in LH canal with pulses of 50-448 $\mu \mathrm{A}$ produces a rightward slow phase eye velocity (negative by convention). (C) Stimulation via electrode E9 in LA canal with pulses of 151-448 $\mu \mathrm{A}$. Right eye response for $396 \mu \mathrm{A}$ stimulus is missing due to video-oculography tracking failure. (D) Dashed lines denote anatomic semicircular canal axes. Solid vectors depict mean rotation axis for each eye during peak excitatory slow phase response eye velocity for electrodes and currents in adjacent legends. Conic sections denote VOR variability via eigenvalue decomposition of the $3 \mathrm{D}$ angular velocity covariance matrix. (E) Canal axes relative to skull landmarks. (F) Same data as $\mathbf{D}$ but viewed from above (i.e., from $+z /$ LHRH axis).

Subject MVI002 produced eye movement responses that increased monotonically with increasing current across all electrodes (Figure 4) except E11, which had high electrical impedance, suggesting wire breakage during implantation. Mean misalignment angle between target canal axis and mean response axis ranged from $0^{\circ}$ to about $40^{\circ}$ for MVI002. The axes of the 2 eyes' responses aligned with each other to within $0^{\circ}$ to about $30^{\circ}$, depending on stimulus current (Supplemental Figure 2). For MVI002, the electrode at the tip of each shank produced significantly larger responses (aligned rank transform [ART] with 1-way repeated-measures ANOVA; $F_{[1,9]}=34.3$, and $P<0.001$ ) than the other 2 electrodes in each canal (Figure $4, \mathrm{~A}-\mathrm{C}$ ). Responses of the other 3 subjects also grew monotonically with current (Supplemental Figure 2).

Initial onset of constant-rate stimulation produced brief yaw and pitch nystagmus but more persistent roll nystagmus, consistent with rapid adaptation driven by retinal slip. Following characterization of responses from all 


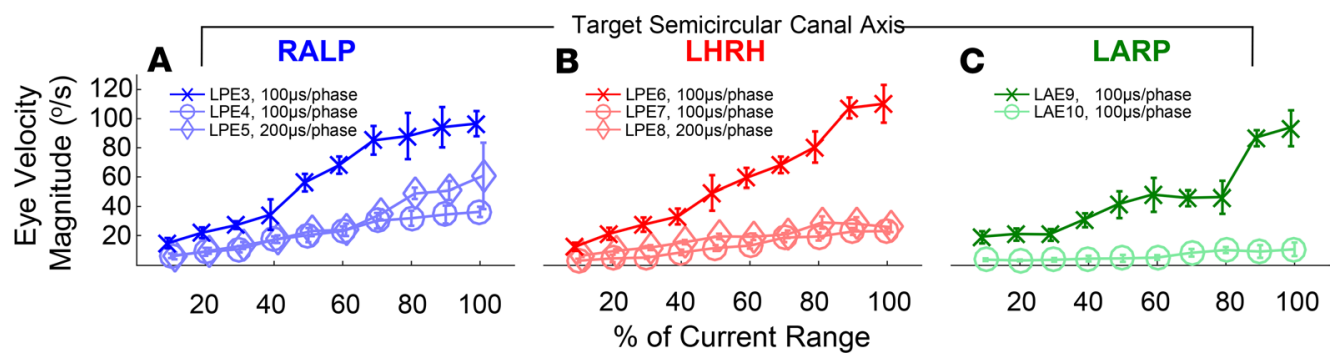

Figure 4. MVIO02 VOR response magnitude depends on electrode location and stimulus intensity. Peak left eye excitatory slow phase velocity component about the target canal's axis as a function of percentage of current amplitude intensity during initial stimulation of subject MVIO02 via all functional electrodes in the left (A) posterior, (B) horizontal, and (C) anterior semicircular canals using 2-Hz-modulated, 40\% duty cycle pulse train alternating between 0 and 200 pps. Each data point depicts mean \pm SD for $n=7-19$ (median 14) cycles. Current intensity $10 \%$ denotes minimum current that evoked a detectable eye movement response; $100 \%$ denotes maximum current tested for that electrode contact and phase duration. E11 not tested due to a high impedance ( $>25 \mathrm{k} \Omega)$. Supplemental Table 3 shows complete mappings of current intensity to pulse amplitude in microamps for each subject. For each ampulla in MVI002, 1 of 3 electrodes clearly outperforms other contacts $700-1100 \mu \mathrm{m}$ away.

stimulating electrodes, 1 electrode per canal was chosen for device activation and long-term use (bold traces in Figure 4 and Supplemental Figure 2 and Supplemental Table 2), and tonic stimulation was initiated on all 3 active electrodes with the subject's head stationary on a bite block. Immediately after stimulation onset and at 5-minute intervals for more than 35 minutes thereafter (black bar at top of Figure 5 and insets A-C), ocular responses were monitored for about 1 minute in darkness. Between those tests in darkness, the subject viewed an Earth-fixed target. For subject MVI002, tonic stimulation onset evoked nystagmus with positive roll (clockwise from the subject's viewpoint), positive pitch (downward), and negative yaw (rightward) slow phase velocity components, consistent with excitation of all 3 left labyrinth canals and LP canal excitation exceeding LA canal excitation. After about 1 minute in darkness, nystagmus was still present but slower. After about 30 minutes of dark/light cycling, the roll nystagmus component in darkness fell to less than $5 \%$ s, a level the yaw and pitch components reached within the first few minutes. Subjects MVI001, MVI003, and MVI004 produced similar results, with stimulation onset eliciting nystagmus that decayed quickly for the yaw and pitch components and within about 30 minutes for the roll component (Supplemental Figure 3). Second-order exponential fits to the nystagmus roll component in darkness yielded dominant time constants of 1.5, 28.2, 7.8, and 9.6 minutes for subjects MVI001, MVI002, MVI003, and MVI004, respectively (RMSE $=3.1 \%$ s, $6.6 \% \mathrm{~s}, 1.7 \%$ s, and $2.5 \%$, respectively).

Prosthetic stimulation remains effective after 8 weeks of continuous use. To assess whether sensitivity to prosthetic stimulation persists in human vestibular implant users despite animal data suggesting that even brief bouts of electrical stimulation engender long-term depression of neural VOR circuits (41), we examined responses after 8 weeks of continuous, $24 \mathrm{~h} / \mathrm{d}$ motion-modulated electrical stimulation. Each subject was tested using sinusoidally modulated prosthetic stimulation while the subject's head was stationary on a bite block in darkness to measure eye movements elicited solely by prosthetic stimulation without contributions from residual natural vestibular function, vision, neck proprioception, or other cues not originating from primary vestibular afferent activity. Each canal's active electrode was modulated alone, while active electrodes in the other canals maintained constant pulse rate and current. Pulse rate and amplitude were modulated over $5 \%-100 \%$ of the dynamic range of pulse rate/pulse amplitude modulation defined by the stimulation parameter map programmed into the subject's PCU (Figure 2, H-K, and Supplemental Table 3).

As was true on the first day of device use, stimulation elicited binocular responses with peak eye velocity magnitude that grew with depth of pulse rate and/or pulse amplitude modulation. For example, Figure 6 shows responses of the left (Figure 6, A-C) and right (Figure 6, D-F) eyes of subject MVI002, for whom stimuli targeting any 1 canal elicited eye movements predominantly aligned with that canal's axis. Similar results were observed for all other subjects (Supplemental Figure 4). These data indicate that although adaptation suppresses nonhuman primate central vestibular neuron sensitivity to prosthetic stimulation within minutes of stimulation onset and suppressed our human subjects' VOR responses within an hour after device activation, prosthetic input remains able to drive eeVOR responses even after 8 weeks of continuous $24 \mathrm{~h} / \mathrm{d}$ stimulation. 


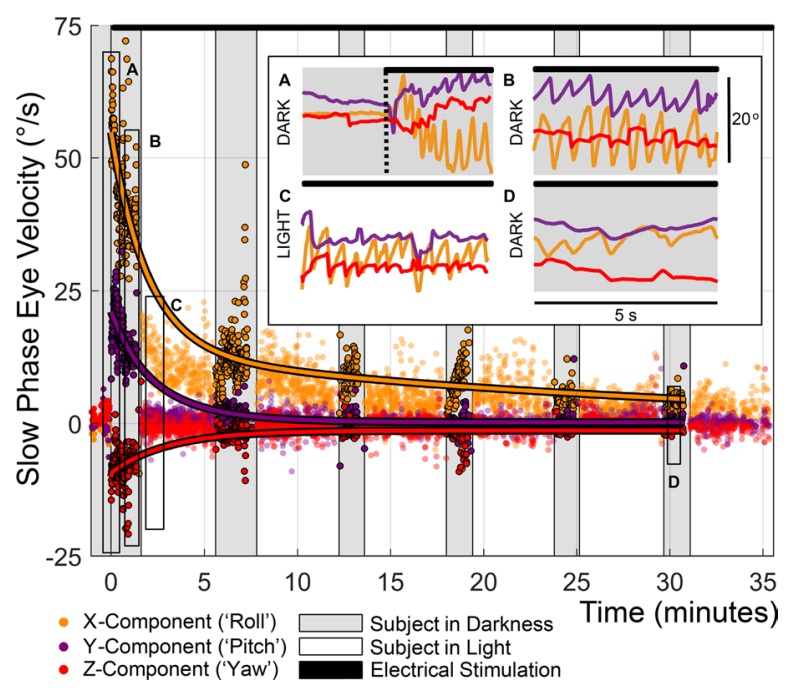

Figure 5. Initial MVI activation elicited robust nystagmus that decayed within $\mathbf{3 0}$ minutes. Three weeks after implantation, continuous electrical stimulation was initiated in subject MVIO02 using at $100 \mathrm{pps} 100 \mu \mathrm{s} / \mathrm{ph}$ ase biphasic, charge-balanced current pulses on electrodes in the $\operatorname{LP}(E 3,599 \mu \mathrm{A})$, horizontal $(E 6,151 \mu \mathrm{A})$ and anterior $(E 9,599 \mu \mathrm{A})$ canals (black bar at top of figure). Eye movements were monitored for 5 -minute cycles of about 1 minute in darkness, then about 4 minutes in light with an Earth-fixed visible target for more than 35 minutes. Each point represents 1 slow phase nystagmus segment. Second-order exponential fits to left eye slow phase velocity in darkness produced dominant time constant estimates of 28.2, 3.33, and 3.0 minutes for roll, pitch, and yaw components, respectively $\left(\mathrm{RMSE}_{x}=6.6^{\circ} / \mathrm{s}, \mathrm{RMSE}_{y}=2.7^{\circ} / \mathrm{s}\right.$, and $\mathrm{RMSE}_{z}=2.7^{\circ} / \mathrm{s}$ ). Insets show $(\mathbf{A})$ robust nystagmus dominated by positive slow phase torsional component at onset of stimulation, (B) extinction of the horizontal nystagmus component in darkness by $t=1$ minutes, (C) suppression of all but the $\sim 18 \%$ torsional response during lights-on testing, and (D) a reduction of the torsional component to $\sim 5^{\circ} / \mathrm{s}$ by $t=30$ minutes.

Concurrent modulation via multiple electrodes encodes $3 D$ head rotational velocity. A vestibular implant intended to provide artificial 3D sensation of head movement should decompose and encode head rotation about any specified head rotation axis by eliciting proportionate activity on the 3 implanted canals' ampullary nerves, which the brainstem subsequently combines to compute an estimate of the head rotation axis. Approximate $3 \mathrm{D}$ vector summation of individual responses driven by multiple canals has been demonstrated in many nonhuman species $(14,26,33)$. To determine whether the same effect can be relied upon when programming a vestibular implant for long-term use in humans, we recorded $3 \mathrm{D}$ eye movements of head-fixed subjects after 8 weeks of continuous motion-modulated stimulation during stimulation via individual canal electrodes (Figure 7A) and then via pairs of electrodes coordinated to represent head rotation about the $+x$ axis (roll axis, parallel to the naso-occipital axis) and $+y$ axis (pitch axis, coincident with the interaural axis). Stimuli were trapezoidally modulated pulse trains (50-ms onset/offset ramp, 150-ms plateau, 250-ms intertrapezoid interval) from $0 \%$ to $50 \%$ modulation depth presented in darkness with the subject's head fixed on a bite block.

Stimulation via subject MVI002's electrodes E6, E9, and E3 individually evoked 3D eye velocities predominantly aligned in each case with the target canal's axis (Figure 7, B-D, respectively). Concurrent excitatory modulation via LP canal electrode E3 and LA canal electrode E9 produced roll eye movements, consistent with the vector sum of responses to excitation via E3 and E9 alone (Figure 7E). Concurrent excitatory modulation via posterior canal electrode E3 and inhibitory downmodulation on posterior canal electrode E9 elicited a downward pitch eye movement, which is positive by the right-hand rule display convention used in Figure 7, F-H. Observations in all other subjects further corroborated the ability of MVI stimulation to encode 3 linearly independent, approximately canal-aligned components of 3D rotational motion, alone and in linear combinations (Supplemental Figure 5 and Supplemental Video 1). The pitch $3 \mathrm{D}$ VOR response in Figure $7 \mathrm{~F}$ demonstrates the importance of acclimating subjects to a tonic baseline level of prosthetic input below which the MVI can downmodulate to decrease neural activity. Prosthetic encoding of a purely pitch head rotation would have been impossible to achieve with a unilateral implant constrained to only increase, not decrease, activity on the left labyrinth's 3 ampullary nerves. By adapting the subject to tonic stimulation and then downmodulating from that baseline to encode inhibition on 1 or 


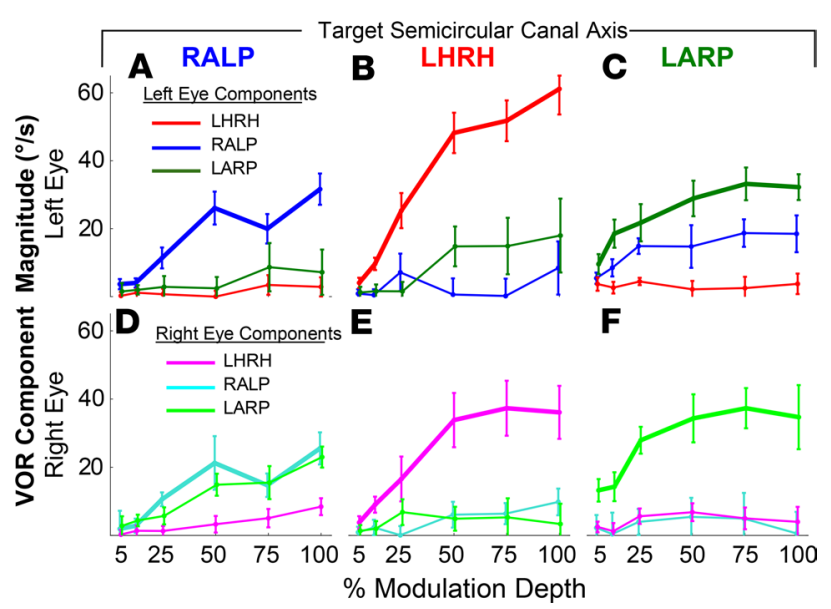

Figure 6. Electrically evoked eye movement responses remain stable after 8 weeks of continuous motion-modulated stimulation. Peak excitatory half-cycle slow phase 3D eye velocity of MVI002's (A-C) left and (D-F) right eyes during stimulus modulation emulating $2-\mathrm{Hz}$ sinusoidal head rotation (with head actually stationary) at modulation depth 5\%-100\% (representing 20-400\% $/ 5$ head velocity) about axes of left posterior (A and D, electrode E3), horizontal (B and $\mathbf{E}, \mathrm{E6}$ ), and anterior ( $\mathbf{C}$ and $\mathbf{F}, \mathrm{E9}$ ) canals, which would ideally drive conjugate (same for both eyes), purely RALP, horizontal LHRH, and LARP responses, respectively. Stimulus parameters are detailed in Supplemental Table 3. Each data point depicts mean \pm SD for $n=9-19$ (median 15) cycles.

more of 3 canals, the MVI can encode head rotations that normally inhibit at least 1 left canal, as is the case for $87.5 \%$ of all possible head rotation directions.

Prosthetic stimulation improves VOR responses to whole-body rotation. To assay system performance during natural rotational stimulation, we measured gain and phase lead of horizontal VOR responses in darkness during $100 \%$ s and $0.1-, 0.2-, 0.5-, 1-$, and 2-Hz whole-body passive sinusoidal rotation before implantation ("preop"), 3 weeks after implantation but before device activation ("postop"), and while using the MVI at each subject's most recent visit (after 812, 738, 782, and 354 days of continuous use for MVI001, MVI002, MVI003, and MVI004, respectively). To isolate the effect of motion-modulated prosthetic stimulation, rotary chair testing was performed with the MVI in its usual mode of operation ("modulation ON"), then repeated on the same day with the device programmed to provide only a constant pulse rate and amplitude ("modulation OFF"), which conveys no motion information apart from falsely signifying that the head is not moving. Complete data sets were obtained for subjects MVI002-MVI004. Having already undergone preoperative rotary chair testing for $0.16,0.32$, and $0.64 \mathrm{~Hz}$ at another institution, yielding VOR gains of 0.01-0.06, MVI001 was not retested at our institution before implantation.

Figure 8 summarizes horizontal VOR gain and phase lead data for MVI002, MVI003, and MVI004, who were tested preop, postop, and during both motion-modulated and constant-rate MVI stimulation at their most recent visits. Data are shown in comparison to normative data for VOR responses to $50 \%$ s whole-body rotary chair rotations reported by Wall et al. for 20 normal subjects of age uniformly distributed over 40-69 years, the age range that encompasses our subjects (49). Supplemental Figure 6 shows data for each subject individually.

All subjects tested preoperatively had abnormally low VOR gain and abnormally high phase lead (i.e., leftward slow phase VOR response velocity leading rightward chair velocity). Postop data reveal a further drop in VOR gain, consistent with electrode implantation worsening native canal function. With the MVI working in its usual operating mode (modulation ON) at the most recent visit, VOR gain was significantly better than preop over all tested frequencies for MVI003 and MVI004, whereas MVI002's gain improved for $0.1-0.5 \mathrm{~Hz}$ but decreased for $1-2 \mathrm{~Hz}$ (ART repeated-measures ANOVA, then Wilcoxon's rank-sum test: $P<0.05$ for every comparison). Modulation ON VOR gain was also significantly better than modulation OFF gain at every frequency for every subject, including MVI001 (Wilcoxon's rank-sum: $P<0.01$ for every comparison). Preop VOR phase lead was abnormally high compared with Wall et al.'s (49) normative data over $0.1-1 \mathrm{~Hz}$ and improved toward normal under the MVI modulation ON test condition. Phase changes at $2 \mathrm{~Hz}$ did not reveal a consistent trend across subjects.

Subjects wear MVI systems $24 \mathrm{~h} / \mathrm{d}$. To the extent that compliance with a course of treatment correlates with a subject's perceived benefit rather than placebo effects or blind adherence to the prescriber's recommendation, 


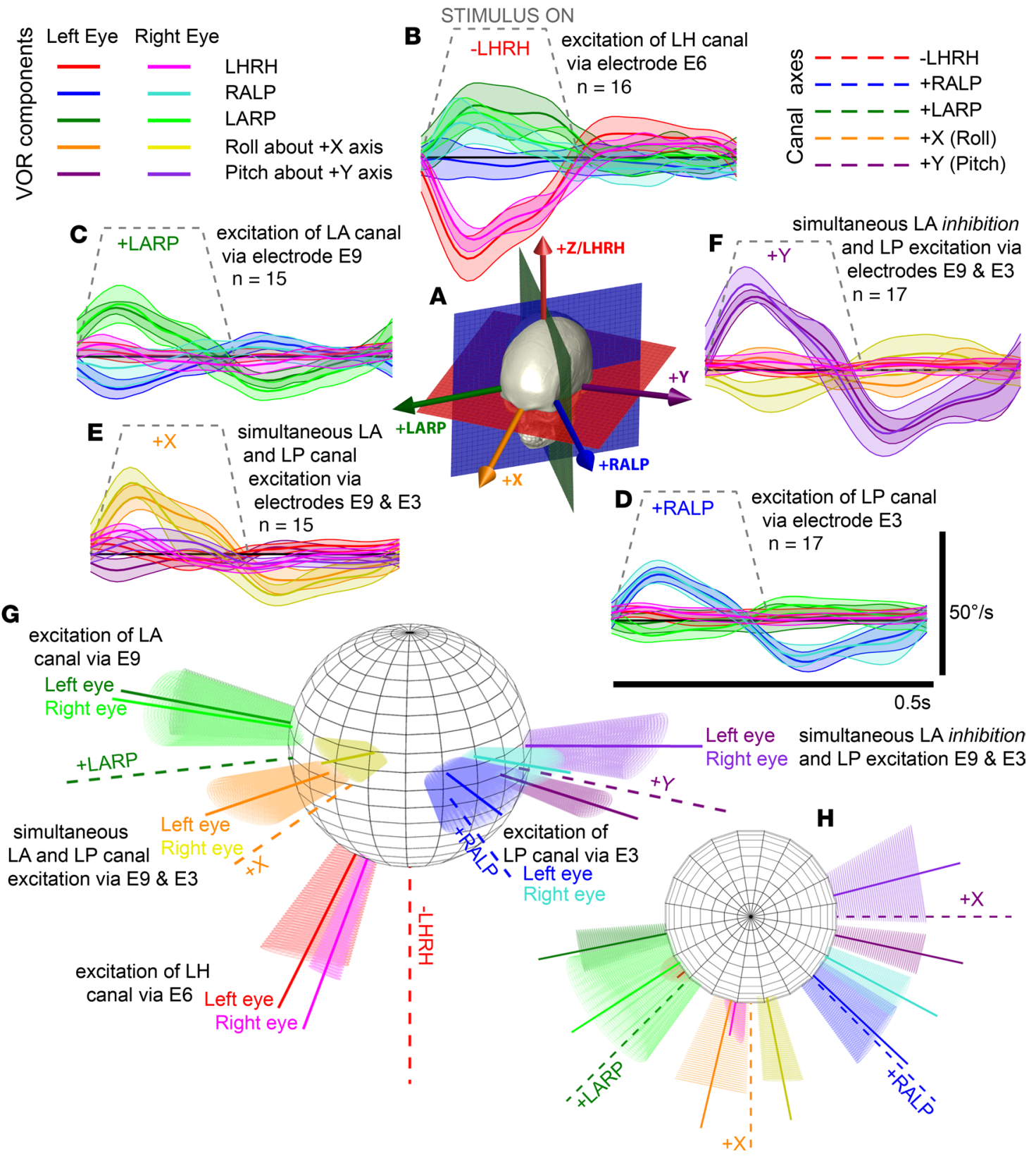

Figure 7. Coordinated multi-electrode input encodes 3D head rotation axis. Simultaneous selective stimulation of multiple ampullary nerves scaled to represent the 3 canal axis components of $3 D$ head rotation can drive 3D VOR responses about an axis that approximates the head rotation axis encoded by the implant. (A) Anatomic canal axes (+LARP, +RALP, + LHRH), naso-occipital $(+x)$ axis, and interaural $(+y)$ axis. Trapezoidal $50 \%$ modulation depth equivalent head velocity stimulation via MVIO02's (B, electrode E6) LH, (C, E9) anterior, and (D, E3) posterior canals individually each evoked 3D eye velocities approximately aligned with target canal axis. Data are shown as mean \pm SD for $n$ cycles. (E) Simultaneous in-phase stimulation via LA E9 and LP E3 yields roll eye response aligned with $+x$ axis, and $(\mathbf{F})$ counter-phase stimulation yields pitch response aligned with $+y$ axis. (C) In each case, mean response axis for each eye aligns approximately with intended head rotation axis as viewed in $3 \mathrm{D}$ (H, same data from top-down view). Elliptical cones illustrate eigenvalues of response axis covariance matrices.

the frequency, consistency, and duration of MVI system use are objective metrics of subjects' perceived benefit. As of when this report was submitted for publication, all 4 subjects had worn their MVI systems continuously, $24 \mathrm{~h} / \mathrm{d}$, for more than 1 year, apart from removal for showering, device maintenance, and experimental testing. Each requested, and the US Food \& Drug Administration (FDA) approved, continued long-term use beyond the end of the originally approved 8-week period of stimulation.

Subjects report more stable vision and decreased postural unsteadiness. Visual acuity during head motion, objective measures of posture and gait, quality-of-life surveys, and other performance metrics are beyond 

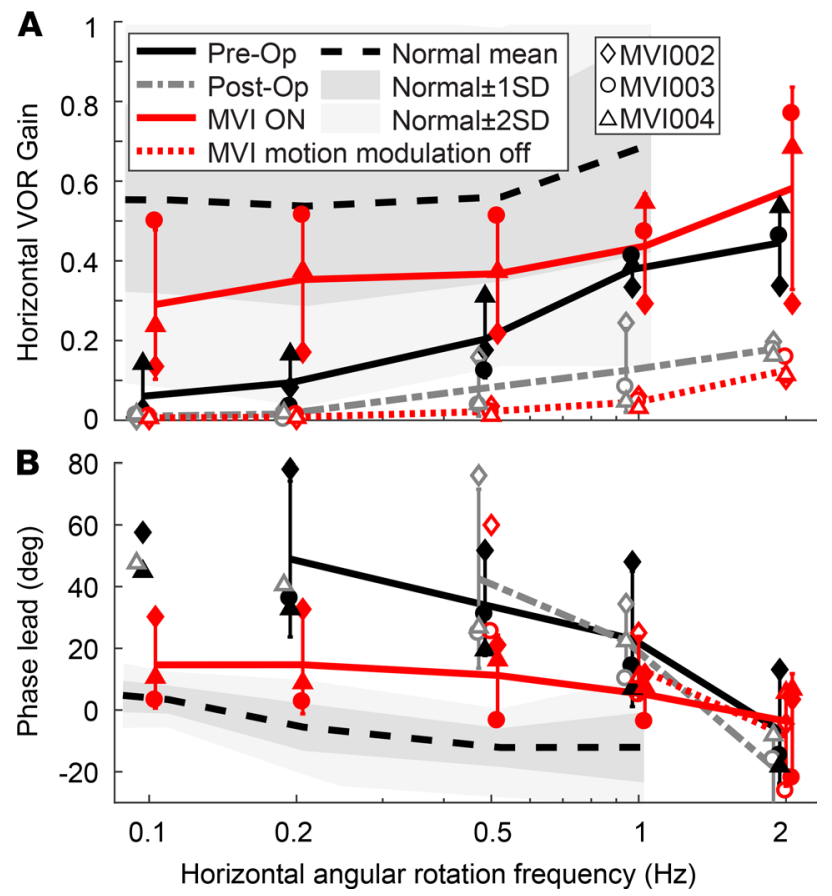

Figure 8. Prosthetic stimulation enhances VOR response to whole-body rotation in darkness. Mean \pm SD horizontal VOR responses to $100 \%$ s peak velocity $0.1-2 \mathrm{~Hz} 100 \%$ s sinusoidal whole-body rotations in darkness on an Earthvertical axis rotary chair were tested for subjects MVI002-MVI004 before surgery (preop); 3 weeks after surgery, just before implant activation (postop); and at the most recent study visit (after 812, 738, 782, and 354 days of continuous stimulation, respectively) with MVI motion modulation on (modulation $\mathrm{ON}$ ) or with a placebo constant-rate stimulus (modulation OFF). VOR (A) gain and (B) phase lead are shown individually for each subject. Each data point is the cycleaveraged mean for $n=2-32$ (median 12.5) cycles. Phase lead is positive when rightward eye velocity leads leftward head velocity and was computed when VOR responses were more than $1.5^{\circ} / \mathrm{s}$. Population mean $\pm \mathrm{SD}$ is shown when data are present for all 3 subjects. Normal mean and ranges within \pm 1 SD and \pm 2 SD are shown for normal 50- to 69-yearold subjects as reported by Wall et al. (49). Supplemental Figure 6 details data individually for each subject, including MVI001, who did not undergo preoperative testing with this paradigm.

the scope of this report; however, the 3D VOR data presented here were corroborated not only by compliance with therapy but also by categorical improvements in subjects' perceived ability to see during head motion and to walk and navigate independently, as objectively evidenced by resumption of activities they had not performed since onset of vestibular hypofunction despite participation in standard-ofcare rehabilitation therapy. Although resumption of such activities might also have been driven in part by placebo effects (such as increased confidence or just a strong desire to perform better after undergoing implant surgery), subjects would have already benefitted from whatever placebo effects accrued from participation in standard-of-care rehabilitation therapy.

Despite being an experienced driver and long-distance runner until his gentamicin-induced vestibular loss 4 years before implantation, subject MVI001 ceased driving entirely after the onset of BVL and avoided sitting in the front of a car because oscillopsia (illusory movement of the visible world during head movement) made the scene through the windshield dizzying. Although still working out daily through weight-lifting and other activities that required little head movement, he had given up outdoor activities, such as jogging, sports, and hunting. He relied on a walking stick during travel. Vestibular rehabilitation exercises provided subjective benefit that plateaued within a year.

At 3 weeks after activation MVI001 reported that his oscillopsia while walking down a long hallway had reduced by $25 \%-50 \%$, he had resumed sitting in the front of vehicles, and he felt able to drive (despite adherence to recommendations against driving while using the MVI). He hiked on sandy terrain, without falling, while hunting for the first time in 4 years. At 3 months after activation, he stopped carrying a walking stick and had resumed treadmill jogging. At 5 months after activation, he ran a $5 \mathrm{~K}$ outdoor race with no falls (though he reported that jogging still caused oscillopsia). He subsequently mastered jumping rope and took up recreational boxing. At 13 months after activation, he reported: "I walked my daughter down the 
aisle and had the father/daughter dance without issue and without a walking stick!! Friends and family were amazed at how well I'm doing." Double-blinded testing to rule out recovery of hair cell function confirmed that under the "modulation OFF" condition his symptoms returned to preoperative levels.

Similarly, subject MVI002 reported that continuous use of the MVI improved his ability to read signs and perceive faces while walking. He reported that, for the first time in years, he felt comfortable walking without focusing downward at his feet. MVI003 transitioned from reliance on furniture, walls, or her husband's arm for balance to walking unaided and traveling independently. MVI004 ceased use of an assistive walking stick and realized her goal of resuming independent travel. All subjects reported that they felt able to resume driving. All requested and were granted FDA approval for long-term use of their vestibular implants beyond the originally planned 8-week trial, and each has used the system continuously since activation.

\section{Discussion}

Binocular 3D oculographic data measured in the first 4 human subjects to receive prolonged, continuous, $24 \mathrm{~h} / \mathrm{d}$ head motion-modulated pulsatile electrical stimulation of all 3 semicircular canal vestibular nerve branches in the Multichannel Vestibular Implant Early Feasibility Study provide strong evidence that prosthetic stimulation using a vestibular implant is a feasible and effective approach to providing artificial sensation of 3D head rotation to individuals disabled by chronic BVL caused by hair cell injury.

Although previous work with animals and human subjects demonstrated the ability to evoke eye movements in response to occasional experimental sessions of electrical stimulation, results of those prior studies raised concerns that continuous, long-term prosthetic stimulation might be thwarted by degradation of electrode-neuron coupling, atrophy of neuronal processes, inability to selectively modulate activity on all 3 ampullary nerves, and/or suppression at central vestibular neuron synapses ( 35 , $39,40)$. The data presented here confirm persistence of electrically evoked eye movement responses after 350-812 days of continuous, $24 \mathrm{~h} / \mathrm{d}$ stimulation. Moreover, whereas prior studies in humans were limited to $2 \mathrm{D}$ video-oculographic measurements, which do not provide sufficient information to accurately determine whether eye movements driven by prosthetic stimulation align with the axis of the targeted canal, the binocular 3D data presented here quantify all 3 canal axis components of the eeVOR response and therefore provide quantitative insight into the relative excitation of each canal. Although we cannot directly assay vestibular afferent fiber activation in human subjects, characterizing evoked eye movements in $3 \mathrm{D}$ rather than $2 \mathrm{D}$ allowed us to estimate relative effects of current spread from each electrode to each of the 3 ampullary nerves. The resulting data are consistent with prior $3 \mathrm{D}$ oculography in animals $(32,33,42)$ and $2 \mathrm{D}$ measurements in humans $(39,40,50-52$, reviewed in ref. 37), but use of binocular 3D oculography revealed disconjugacy inconsistent with the expected effects of purely selective ampullary nerve stimulation. The disconjugate eye movements we observed may reflect stimulus current spreading to the utricle and/or saccule.

Does current targeting ampullary nerves spread to the utricle or saccule? Isolated mechanical stimulation of 1 or more ampullary nerves, without modulation of utricular or saccular nerve activity, produces binocular vestibulo-ocular responses that are conjugate in 3D (i.e., similar in 3D axis and speed for the left and right eyes) and characterized by an angular velocity component that fades within a few minutes $(52,53)$. In contrast, natural modulation of utricular and/or saccular nerve activity, as occurs during a static head tilt, centrifugal stimulation, or translational head acceleration, can elicit disconjugate eye movements, persistent eye position deviation, and perception of head tilt or translation (54). Disconjugacy, static eye deviation, and head tilt or translation percepts can therefore serve as indicators of utricular and/or saccular activity in a stationary test subject instructed not to voluntarily activate vergence, eccentric gaze, or other nonvestibular oculomotor systems that elicit disconjugate and static eye deviations.

In the present study, prosthetic stimulation targeting ampullary nerves typically produced approximately conjugate nystagmus eye movements without static deviation of eye angular position or perception of tilt or translation. This finding supports a conclusion that prosthetic stimulation via electrodes in the ampullae can effectively drive canal-ocular reflexes while avoiding significant activation of utricular- or saccular-ocular reflexes. However, if intense enough to modulate activity in the utricular or saccular nerves, current spread from ampullary electrodes should drive otolith-ocular reflexes that drive disconjugate eye movements. Examples of this were observed in MVI001 (Supplemental Figure 1, A and C) and MVI002 (Figure 3, A and C), for whom stimulation via electrodes in the anterior and posterior canals elicited disconjugate eye movements not only during the initial testing but also after 8 weeks 
of continuous motion-modulated stimulation (Figure 6 and Supplemental Figure 4). Interestingly, the pattern of disconjugacy was both electrode and eye dependent. For example, stimulation via MVI002's LP canal electrode elicited left eye movement aligned with the RALP plane, but right eye movement more nearly aligned with the pitch axis (Figure 3A and Figure 6A). An analogous but opposite pattern was observed during electrical stimulation via an electrode in the LA canal (Figure 3C and Figure 6C). The physiologic basis for this pattern, which was consistently observed in MVI001 and MVI002 but not in the other 2 subjects, is unclear. Video-oculography system artifact was excluded because calibration recordings made during LARP, RALP, and LHRH head rotations with 3 normal subjects viewing an Earth-stationary full-field distant scene in light yielded conjugate eye movements with a mean angle between the left and right eye $3 \mathrm{D}$ rotational velocity axes of $9.0 \pm 6.7^{\circ}, 8.1 \pm 6.1^{\circ}$, and $9.9 \pm 9.3^{\circ}$ for velocities at least $5^{\circ} / \mathrm{s}$ and $7.2 \pm 4.3^{\circ}, 6.5 \pm 4.1^{\circ}$, and $7.8 \pm 6.0^{\circ}$ for all measured velocities at least $20^{\circ} / \mathrm{s}$. Those calibration data reflect measurement noise, are all similar to misalignment angles measured using $3 \mathrm{D}$ scleral coils in normal humans (55) and during single-canal mechanical stimulation (53), and are significantly less than the $30.5 \pm 10.2^{\circ}$ evident in Figure 6C (unpaired $t$ test: $P<0.001$ for every comparison). It seems likely that disconjugacy was caused by spurious utricular and/or saccular activation; however, the pattern of utricular/saccular activity that would elicit such eye movement responses is not obvious. Another possibility is that a latent esotropia, which was observed in subject MVI002, caused convergence of the eyes during testing in darkness. Previous work investigating the influence of initial binocular 3D position on the angular VOR showed that vergence leads to disconjugate responses during natural stimulation of the posterior and anterior canals (56).

Subjects adapt quickly after initial onset of continuous MVI stimulation. All components of the slow phase $3 \mathrm{D}$ VOR response to sudden onset of tonic prosthetic vestibular stimulation decayed to less than $5 \%$ s within about 30 minutes for every subject (Figure 5 and Supplemental Figure 3), with yaw and pitch decaying much faster than roll. These data support the hypothesis that retinal slip error caused by roll nystagmus does not drive compensatory VOR adaptation as effectively as retinal slip due to horizontal and vertical nystagmus components because the former tend not to displace a fixation target from the retinal foveae whereas the latter do (57-60). They are also consistent with previously reported data acquired using $2 \mathrm{D}$ video-oculography $(38,39,61$, reviewed in ref. 37). In subjects MVI003 and MVI004, prosthetic stimulation onset evoked a yaw nystagmus component of about $-3 \%$ s that reversed direction during the first minute (Figure 5, B and C, respectively). This may represent a nystagmus reversal analogous to that observed for normal subjects during prolonged constant-velocity whole-body rotation (62-66). The data also indicate that even the roll/torsional component of nystagmus adapts to a low enough level over a short enough time after activation that it should eventually be feasible to accomplish an initial vestibular implant programming session within about 2 hours (the typical duration of an initial cochlear implant activation visit at Johns Hopkins), if 3D eeVOR video-oculography data analysis can be sufficiently automated.

All subjects reported briefly intense vertigo for less than 5 minutes immediately upon onset of stimulation, and all described perception of yaw and roll head movement, consistent with the expected vector sum of roughly equal components about the axes of the 3 implanted/excited canals. The motion percept faded rapidly and ended less than 10 min after onset of stimulation. Starting about 35 minutes after stimulation onset, each subject was able to walk around the clinic, initially assisted but then completely independently less than 60 minutes after stimulation onset. Each subject was discharged from the outpatient clinic on the activation day, without requiring overnight inpatient observation, parenteral medications for treatment of vertigo, or other costly interventions that would otherwise pose financial barriers to broad adoption of vestibular implantation into clinical practice.

Directional plasticity. In comparison with the other 2 subjects, MVI003 and MVI004 produced more misaligned responses during stimulation via LH canal electrode E6 on the first day of testing (Supplemental Figure 1, $\mathrm{H}$ and L). Even after 8 weeks of adaptation to continuous motion-modulated stimulation, MVI004's responses to stimulation via E6 included a prominent LARP component (Supplemental Figure 4C). Previous studies in chinchillas (28) and nonhuman primates (32) showed that VOR directional plasticity can rapidly and significantly minimize off-axis VOR components over the first 7 days of continuous, motion-modulated prosthetic stimulation, presumably via the same sort of neuronal learning mechanisms that reorient VOR direction when a normal subject views the world through prisms or other optical manipulations that cause the visual scene to rotate about an axis other than the head rotation axis (67-70). In this study, MVI004's responses did not show this cross-axis adaptation effect. One potential reason could be that plasticity effects occurred but plateaued before the 1-week 
post-activation assessment. Differences in stimulus-encoding scheme may also have played a role. Our animal studies of directional plasticity used pulse rate modulation of constant current amplitude stimulus pulses to encode head rotations $(28,32)$. In contrast, subject MVI004's device was programmed to modulate both pulse rate and current amplitude, with the latter possibly causing sufficient current spread to dynamically alter the relative activity in different vestibular nerve branches with head motion in a way that interfered with neuronal circuits that normally mediate directional plasticity.

Study limitations. All subjects described herein suffered BVL due to apparent ototoxic injury after administration of aminoglycoside antibiotics. We started with this well-defined group because we could be reasonably sure that bypassing dysfunctional hair cells through electrical stimulation of primary vestibular afferent neurons would be effective. Whether favorable outcomes can be achieved in individuals with idiopathic vestibular areflexia cannot be predicted from these data with high confidence because the site of dysfunction in idiopathic cases could be downstream from the vestibular nerve. Unlike sensorineural hearing loss, for which otoacoustic emissions provide a direct, specific clinical assay of cochlear health, there is no test of vestibular labyrinth sensation that is independent of central nervous system circuits and/ or downstream effectors, such as extraocular and postural control muscles.

Another limitation of the present study is that stimulation parameters were set and adjusted over time on a subject-by-subject, canal-by-canal, and electrode-by-electrode basis, with each subject's stimulus parameters adjusted at least once in an effort to identify his/her optimal settings (Supplemental Tables 3 and 4). This constrains our ability to combine and generalize results across subjects, canals, and electrodes. The data presented here should therefore be interpreted as outcomes of an intention-to-treat study (71), with the intended treatment in this case including adjustments of device settings similar to the routine clinical practice of adjusting cochlear implant stimulus parameters over time. Holding stimulus parameters constant throughout the assessment period would have offered a cleaner assay of how VOR responses evolve over time independent of such interventions, but the ethical imperative to seek the best possible outcome for each study participant required a trade-off between enforcing consistency and striving for the best achievable clinical outcome.

Eye movement response velocities evoked in human subjects were small compared with those of nonhuman primates (30-35), chinchillas (25-29), and guinea pigs (19-23). The cause of this interspecies disparity, which has been noted in prior studies $(37,39)$, is unclear. Rodents typically have lower natural VOR gains than humans, while the natural VOR of nonhuman primates closely approximates ours (72). Species-specific differences in sensitivity to aminoglycoside antibiotics could play a role, as could differences in gentamicin dose, route of delivery to the labyrinth, subject age, and duration since the onset of ototoxic injury. However, adult nonhuman primates treated with gentamicin at doses sufficient to permanently wipe out natural VOR responses can still have robust electrically evoked 3D VOR responses years later, despite postmortem histologic exam revealing absence of type I hair cells and absence of stereocilia on type II hair cells (12). Human subjects in this study may have had even more severe hair cell lesions, but the presence of some natural VOR responses to whole-body rotation preoperatively suggests that they had at least some hair cell and afferent neuron survival. Identifying key determinants that result in nonhuman primates having stronger eeVOR responses compared with our human subjects may reveal paths to improving clinical vestibular implant performance.

We implanted the single reference electrode in the common crus based on favorable results of experiments in nonhuman primates using that approach (30-33). Placing it elsewhere might yield more effective stimulation. As a modified cochlear implant with 12 stimulating electrodes but a single stimulation reference electrode, the MVI's implanted stimulator cannot perform bipolar stimulation (i.e., between 2 adjacent electrodes within 1 ampulla), which should achieve more focal stimulation.

The implanted stimulator relies on power and signals transmitted from the external HWU, which is susceptible to motion relative to the skull and decoupling from the scalp. Every subject experienced occasional HWU decoupling, typically while sleeping. Incorporating a motion sensor and battery in the implanted stimulator to make it autonomous would enhance stimulus fidelity and reliability.

Reports of changes in symptoms must always be considered in the context of potential for placebo effects or other biases. For example, hoping for symptom improvement could bias subjects toward experiencing and reporting it. However, the VOR data presented here are objective measures of prosthetic stimulation efficacy, as is resumption of balance-dependent activities that a subject did not perform before implantation despite standard-of-care treatment, such as MVI001's resumption of treadmill jogging. 


\section{Methods}

Study design. The Multichannel Vestibular Implant Early Feasibility Study (ClinicalTrials.gov: NCT02725463) is a first-in-human, nonrandomized, self-controlled, longitudinal early clinical feasibility study to assess safety and tolerability of the Labyrinth Devices MVI as a treatment for BVL in a convenience sample of adults with symptoms and signs of chronic BVL. Each subject served as his/her own control. The focus of the present report is characterization of electrically evoked 3D VOR responses with the head held fixed on a bite block, a condition in which eye movement responses temporally correlated with prosthetic input can be attributed to that input because any residual natural mechanosensory function in the subject's semicircular canals should report an absence of head motion. Complementing those data are responses during wholebody rotation in darkness about an Earth-vertical axis with prosthetic input that is either motion modulated or constant rate. The former is the implant's normal operating mode; the latter is a control condition that assays responses to residual natural horizontal canal mechanosensation.

Details of methods are presented in the Supplemental Methods.

Statistics. All data are reported as mean \pm SD. Tests of normality indicated most data residuals significantly deviated from a normal distribution (Anderson-Darling test, 95\% CI). We used the ART procedure (73-75) to perform a nonparametric multifactorial repeated-measures ANOVA and evaluate differences in evoked responses across each stimulus condition. This was typically performed with factors of MVI stimulator condition ("preoperative," "postoperative and prestimulation," "modulation ON," and "modulation OFF") and frequency (0.1-2 Hz). Post hoc pairwise comparisons were made using 2-sided Wilcoxon's rank-sum tests. Statistical significance for all tests was set at $P<0.05$.

Study approval. This study was conducted under a protocol approved by the JHU Institutional Review Board (IRB study number NA_00051349) and registered on the ClinicalTrials.gov database (NCT02725463). The MVI system used in this study was developed and manufactured through a collaboration between Labyrinth Devices, LLC, and Med-El GmbH. The devices were provided by Labyrinth Devices, LLC, to the JHU study team for use only in this study under FDA Investigational Device Exemption G150198. Informed consent was obtained after the nature and possible consequences of the studies were explained. Written informed consent was also obtained for all subject images in this manuscript.

\section{Author contributions}

PJB, DPS, MR, NSV, MRC, AIA, BJM, AH, AMR, AM, RD, GYF, NSD, BKW, CT, SPB, DCR, KEL, YG, MCS, JPC, AJ, and CCDS contributed to experiment design and read and approved the manuscript. PJB collected and analyzed data, generated figures, and drafted the manuscript. DPS, MR, NSV, MRC, AIA, BJM, and KEL assisted with data collection and study coordination. MR, NSV, and NSD contributed to MVI system component development and test equipment development at Labyrinth Devices, LLC. AH, AMR, AM, RD, and AJ contributed to MVI system development at Med-El GmbH, where AJ served as project leader. CCDS, as principal investigator of Vestibular NeuroEngineering Laboratory and recipient of R01DC13536, contributed significantly to all aspects of this work; performed the surgical implantations; contributed to experiment design, data collection, data analysis, and manuscript generation; and supervised aspects of this work performed by Labyrinth Devices, LLC. In accordance with JHU policies on conflict of interest, JPC served as nonconflicted principal investigator of study protocol NA_00051349 and participated in candidacy reviews, implantation surgeries, data integrity reviews, and editing this manuscript.

\section{Acknowledgments}

The authors thank the study subjects for their courage, patience, and numerous hours devoted to these experiments; Johns Hopkins Hospital and School of Medicine staff for assistance in subject care; and members of the study's data safety management board, David Eisenman, Frank Lin, and Charles Matthew Stewart. Figures 1 and 2 are reproduced by permission from Labyrinth Devices, LLC, C2019. The study was funded by NIH/National Institute on Deafness and Other Communication Disorders R01DC013536 and 2T32DC000023; Labyrinth Devices, LLC; and Med-E1 GmbH.

Address correspondence to: Charles C. Della Santina, 601 North Caroline Street, Baltimore, Maryland 21287, USA. Phone: 410.502.8047; Email: cds@jhmi.edu. 
1. Ward BK, Tarnutzer AA. Editorial: bilateral vestibulopathy - current knowledge and future directions to improve its diagnosis and treatment. Front Neurol. 2018;9:762.

2. Whitney SL, Sparto PJ, Cook JR, Redfern MS, Furman JM. Symptoms elicited in persons with vestibular dysfunction while performing gaze movements in optic flow environments. $J$ Vestib Res. 2013;23(1):51-60.

3. Sun DQ, Ward BK, Semenov YR, Carey JP, Della Santina CC. Bilateral vestibular deficiency: quality of life and economic implications. JAMA Otolaryngol Head Neck Surg. 2014;140(6):527-534.

4. Ward BK, Agrawal Y, Hoffman HJ, Carey JP, Della Santina CC. Prevalence and impact of bilateral vestibular hypofunction: results from the 2008 US National Health Interview Survey. JAMA Otolaryngol Head Neck Surg. 2013;139(8):803-810.

5. Inoue A, et al. Effect of vestibular dysfunction on the development of gross motor function in children with profound hearing loss. Audiol Neurootol. 2013;18(3):143-151.

6. Wilson BS. The modern cochlear implant: a triumph of biomedical engineering and the first substantial restoration of human sense using a medical intervention. IEEE Pulse. 2017;8(2):29-32.

7. Advanced Bionics LLC. 2019 Global Implant Reliability Report. https://advancedbionics.com/content/dam/advancedbionics/Documents/Global/en_ce/Professional/Technical-Reports/Reliability/027-N025-02-RevC\%20Reliability\%20Report\%20 2019\%20Cochlear\%20Implant_RS.pdf. Accessed October 29, 2019.

8. Cochlear Limited. Cochlear Limited 2019 Annual Report. https://www.cochlear.com/c782c6e8-cd66-402e-87b8-936db9f1 fea8/2019AnnualReport.pdf?MOD=AJPERES\&amp;CONVERT_TO=url\&amp;CACHEID=ROOTWORKSPACE-c782c6 e8-cd66-402e-87b8-936db9f1 fea8-mOnUHj-. Accessed October 24, 2019.

9. Goldberg JM. The vestibular end organs: morphological and physiological diversity of afferents. Curr Opin Neurobiol. 1991;1(2):229-235.

10. Carey JP, Della Santina C. Principles of applied vestibular physiology. In Flint PW et al., eds.: Cummings Otolaryngology - Head and Neck Surgery. 5th ed. Philadelphia, Pennsylvania, USA; Elsevier:2010;2276-2304.

11. Strupp M, Feil K, Dieterich M, Brandt T. Bilateral vestibulopathy. Handb Clin Neurol. 2016;137:235-240.

12. Sun DQ, et al. Histopathologic changes of the inner ear in rhesus monkeys after intratympanic gentamicin injection and vestibular prosthesis electrode array implantation. J Assoc Res Otolaryngol. 2015;16(3):373-387.

13. Breuer J. Neue Versuche an den Ohrbogengängen. Arch Physiol. 1889;44(S):135-152.

14. Cohen B, Suzuki JI, Bender MB. Eye movements from semicircular canal nerve stimulation in the cat. Ann Otol Rhinol Laryngol. 1964;73:153-169.

15. Suzuki JI, Cohen B. Head, eye, body and limb movements from semicircular canal nerves. Exp Neurol. 1964;10:393-405.

16. Suzuki JI, Goto K, Tokumasu K, Cohen B. Implantation of electrodes near individual vestibular nerve branches in mammals. Ann Otol Rhinol Laryngol. 1969;78(4):815-826.

17. Ezure K, Cohen MS, Wilson VJ. Response of cat semicircular canal afferents to sinusoidal polarizing currents: implications for input-output properties of second-order neurons. J Neurophysiol. 1983;49(3):639-648.

18. Suzuki JI, Cohen B, Bender MB. Compensatory eye movements induced by vertical semicircular canal stimulation. Exp Neurol. 1964;9:137-160

19. Gong W, Merfeld DM. Prototype neural semicircular canal prosthesis using patterned electrical stimulation. Ann Biomed Eng. 2000;28(5):572-581

20. Gong W, Merfeld DM. System design and performance of a unilateral horizontal semicircular canal prosthesis. IEEE Trans Biomed Eng. 2002;49(2):175-181.

21. Merfeld DM, Gong W, Morrissey J, Saginaw M, Haburcakova C, Lewis RF. Acclimation to chronic constant-rate peripheral stimulation provided by a vestibular prosthesis. IEEE Trans Biomed Eng. 2006;53(11):2362-2372.

22. Saginaw MA, Gong W, Haburcakova C, Merfeld DM. Attenuation of eye movements evoked by a vestibular implant at the frequency of the baseline pulse rate. IEEE Trans Biomed Eng. 2011;58(10):2732-2739.

23. Lewis RF, Nicoucar K, Gong W, Haburcakova C, Merfeld DM. Adaptation of vestibular tone studied with electrical stimulation of semicircular canal afferents. J Assoc Res Otolaryngol. 2013;14(3):331-340.

24. Gorgiladze GI. Electrical stimulation of labyrinths and vestibular reactions. Bull Exp Biol Med. 2004;138(6):629-631.

25. Della Santina CC, Migliaccio AA, Patel AH. A multichannel semicircular canal neural prosthesis using electrical stimulation to restore 3-d vestibular sensation. IEEE Trans Biomed Eng. 2007;54(6 Pt 1):1016-1030.

26. Fridman GY, Davidovics NS, Dai C, Migliaccio AA, Della Santina CC. Vestibulo-ocular reflex responses to a multichannel vestibular prosthesis incorporating a 3D coordinate transformation for correction of misalignment. $J$ Assoc Res Otolaryngol. 2010;11(3):367-381.

27. Davidovics NS, Fridman GY, Chiang B, Della Santina CC. Effects of biphasic current pulse frequency, amplitude, duration, and interphase gap on eye movement responses to prosthetic electrical stimulation of the vestibular nerve. IEEE Trans Neural Syst Rehabil Eng. 2011;19(1):84-94

28. Dai C, et al. Cross-axis adaptation improves 3D vestibulo-ocular reflex alignment during chronic stimulation via a head-mounted multichannel vestibular prosthesis. Exp Brain Res. 2011;210(3-4):595-606.

29. Davidovics NS, Fridman GY, Della Santina CC. Co-modulation of stimulus rate and current from elevated baselines expands head motion encoding range of the vestibular prosthesis. Exp Brain Res. 2012;218(3):389-400.

30. Chiang B, Fridman GY, Dai C, Rahman MA, Della Santina CC. Design and performance of a multichannel vestibular prosthesis that restores semicircular canal sensation in rhesus monkey. IEEE Trans Neural Syst Rehabil Eng. 2011;19(5):588-598.

31. Dai C, Fridman GY, Davidovics NS, Chiang B, Ahn JH, Della Santina CC. Restoration of 3D vestibular sensation in rhesus monkeys using a multichannel vestibular prosthesis. Hear Res. 2011;281(1-2):74-83.

32. Dai C, et al. Directional plasticity rapidly improves $3 \mathrm{D}$ vestibulo-ocular reflex alignment in monkeys using a multichannel vestibular prosthesis. J Assoc Res Otolaryngol. 2013;14(6):863-877.

33. Davidovics NS, Rahman MA, Dai C, Ahn J, Fridman GY, Della Santina CC. Multichannel vestibular prosthesis employing modulation of pulse rate and current with alignment precompensation elicits improved VOR performance in monkeys. $J$ Assoc Res Otolaryngol. 2013;14(2):233-248.

34. Nie K, et al. An experimental vestibular neural prosthesis: design and preliminary results with rhesus monkeys stimulated with 
modulated pulses. IEEE Trans Biomed Eng. 2013;60(6):1685-1692.

35. Phillips C, et al. Longitudinal performance of an implantable vestibular prosthesis. Hear Res. 2015;322:200-211.

36. Wall C, Kos MI, Guyot JP. Eye movements in response to electric stimulation of the human posterior ampullary nerve. Ann Otol Rhinol Laryngol. 2007;116(5):369-374.

37. Guyot JP, Perez Fornos A. Milestones in the development of a vestibular implant. Curr Opin Neurol. 2019;32(1):145-153.

38. Golub JS, et al. Prosthetic implantation of the human vestibular system. Otol Neurotol. 2014;35(1):136-147.

39. Phillips JO, et al. Vestibular implantation and longitudinal electrical stimulation of the semicircular canal afferents in human subjects. J Neurophysiol. 2015;113(10):3866-3892.

40. Mitchell DE, Della Santina CC, Cullen KE. Plasticity within non-cerebellar pathways rapidly shapes motor performance in vivo. Nat Commun. 2016;7:11238.

41. Mitchell DE, Della Santina CC, Cullen KE. Plasticity within excitatory and inhibitory pathways of the vestibulo-spinal circuitry guides changes in motor performance. Sci Rep. 2017;7(1):853.

42. Dai C, Fridman GY, Davidovics NS, Chiang B, Ahn JH, Della Santina CC. Restoration of 3D vestibular sensation in rhesus monkeys using a multichannel vestibular prosthesis. Hear Res. 2011;281(1-2):74-83.

43. Sadeghi SG, Minor LB, Cullen KE. Response of vestibular-nerve afferents to active and passive rotations under normal conditions and after unilateral labyrinthectomy. J Neurophysiol. 2007;97(2):1503-1514.

44. Otero-Millan J, Roberts DC, Lasker A, Zee DS, Kheradmand A. Knowing what the brain is seeing in 3 dimensions: A novel, noninvasive, sensitive, accurate, and low-noise technique for measuring ocular torsion. $J$ Vis. 2015;15(14):11.

45. Migliaccio AA, Todd MJ. Real-time rotation vectors. Australas Phys Eng Sci Med. 1999;22(2):73-80.

46. Della Santina CC, Potyagaylo V, Migliaccio AA, Minor LB, Carey JP. Orientation of human semicircular canals measured by 3-dimensional multiplanar CT reconstruction. J Assoc Res Otolaryngol. 2005;6(3):191-206.

47. Haustein W. Considerations on Listing's Law and the primary position by means of a matrix description of eye position control. Biol Cybern. 1989;60(6):411-420.

48. Tan GX, et al. Automated head motion system improves reliability and lessens operator dependence for head impulse testing of vestibular reflexes. IEEE Int Symp Med Meas Appl. 2017;2017:94-99.

49. Wall C, Black FO, Hunt AE. Effects of age, sex and stimulus parameters upon vestibulo-ocular responses to sinusoidal rotation. Acta Otolaryngol. 1984;98(3-4):270-278.

50. Wall C, Kos MI, Guyot JP. Eye movements in response to electric stimulation of the human posterior ampullary nerve. Ann Otol Rhinol Laryngol. 2007;116(5):369-374.

51. van de Berg R, et al. The vestibular implant input interacts with residual natural function. Front Neurol. $2017 ; 8: 644$.

52. Cremer PD, et al. Posterior semicircular canal nystagmus is conjugate and its axis is parallel to that of the canal. Neurology. 2000;54(10):2016-2020.

53. Cremer PD, Minor LB, Carey JP, Della Santina CC. Eye movements in patients with superior canal dehiscence syndrome align with the abnormal canal. Neurology. 2000;55(12):1833-1841.

54. Leigh RJ, Zee DS. The Neurology of Eye Movements. Oxford, United Kingdom; Oxford University Press:2015

55. Aw ST, Haslwanter T, Halmagyi GM, Curthoys IS, Yavor RA, Todd MJ. Three-dimensional vector analysis of the human vestibuloocular reflex in response to high-acceleration head rotations. I. Responses in normal subjects. J Neurophysiol. 1996;76(6):4009-4020.

56. Migliaccio AA, Della Santina CC, Carey JP, Minor LB, Zee DS. The effect of binocular eye position and head rotation plane on the human torsional vestibuloocular reflex. Vision Res. 2006;46(16):2475-2486.

57. Berthoz A, Jones GM, Bégué AE. Differential visual adaptation of vertical canal-dependent vestibulo-ocular reflexes. Exp Brain Res. 1981;44(1):19-26.

58. Berthoz A., Jones G.M., Bégué A. Long-Term Effects of Dove Prism Vision on Torsional VOR and Head-Eye Coordination. In Flohr H., Precht W, eds. Lesion-Induced Neuronal Plasticity in Sensorimotor Systems. Berlin;Springer:1981:277-283.

59. Solomon D, Zee DS, Straumann D. Torsional and horizontal vestibular ocular reflex adaptation: 3-dimensional eye movement analysis. Exp Brain Res. 2003;152(2):150-155.

60. Trillenberg P, Shelhamer M, Roberts DC, Zee DS. Cross-axis adaptation of torsional components in the yaw-axis vestibulo-ocular reflex. Exp Brain Res. 2003;148(2):158-165.

61. Guyot JP, Sigrist A, Pelizzone M, Kos MI. Adaptation to steady-state electrical stimulation of the vestibular system in humans. Ann Otol Rhinol Laryngol. 2011;120(3):143-149.

62. Furman JM, Koizuka I, Schor RH. Characteristics of secondary phase post-rotatory nystagmus following off-vertical axis rotation in humans. J Vestib Res. 2000;10(3):143-150.

63. Furman JM, Hain TC, Paige GD. Central adaptation models of the vestibulo-ocular and optokinetic systems. Biol Cybern. 1989;61(4):255-264.

64. Goldberg JM, Fernandez C. Physiology of peripheral neurons innervating semicircular canals of the squirrel monkey. I. Resting discharge and response to constant angular accelerations. J Neurophysiol. 1971;34(4):635-660.

65. Leigh RJ, Robinson DA, Zee DS. A hypothetical explanation for periodic alternating nystagmus: instability in the optokinetic-vestibular system. Ann N Y Acad Sci. 1981;374:619-635.

66. Malcolm R, Jones GM. A quantitative study of vestibular adaptation in humans. Acta Otolaryngol. 1970;70(2):126-135.

67. Schultheis LW, Robinson DA. Directional plasticity of the vestibuloocular reflex in the cat. Ann N Y Acad Sci. 1981;374:504-512.

68. Gonshor A, Jones GM. Short-term adaptive changes in the human vestibulo-ocular reflex arc. J Physiol (Lond). 1976;256(2):361-379.

69. Gonshor A, Jones GM. Extreme vestibulo-ocular adaptation induced by prolonged optical reversal of vision. J Physiol (Lond). $1976 ; 256(2): 381-414$.

70. Gonshor A, Jones GM. Postural adaptation to prolonged optical reversal of vision in man. Brain Res. 1980;192(1):239-248.

71. Gupta SK. Intention-to-treat concept: a review. Perspect Clin Res. 2011;2(3):109-112.

72. Migliaccio AA, Minor LB, Della Santina CC. Adaptation of the vestibulo-ocular reflex for forward-eyed foveate vision. J Physiol (Lond). 2010;588(Pt 20):3855-3867.

73. Salter KC, Fawcett RF. The art test of interaction: a robust and powerful rank test of interaction in factorial models. Commun 
Stat Simul Comput. 1993;22(1):137-153.

74. Zhuang Y, Guan Y, Qiu L, Lai M, Tan MT, Chen P. A novel rank-based non-parametric method for longitudinal ordinal data. Stat Methods Med Res. 2018;27(9):2775-2794.

75. Wobbrock JO, Findlater L, Gergle D, Higgins JJ. The aligned rank transform for nonparametric factorial analyses using only ANOVA procedures. Proc SIGCHI Conf Hum Factors Comput Syst. 2011;143-146.

76. Du SX, Du YF, Wu XL. [Detection of dissolved organic matter based on 3-dimensional first-order derivative fluorescence spectrometry]. Guang Pu Xue Yu Guang Pu Fen Xi. 2010;30(12):3268-3271.

77. Della Santina CC, Potyagaylo V, Migliaccio AA, Minor LB, Carey JP. Orientation of human semicircular canals measured by 3-dimensional multiplanar CT reconstruction. J Assoc Res Otolaryngol. 2005;6(3):191-206.

78. Haslwanter T. Mathematics of 3-dimensional eye rotations. Vision Res. 1995;35(12):1727-1739.

79. Sadeghi SG, Minor LB, Cullen KE. Response of vestibular-nerve afferents to active and passive rotations under normal conditions and after unilateral labyrinthectomy. J Neurophysiol. 2007;97(2):1503-1514. 